\title{
Risk Factors for Recurrent Pancreatitis After First Episode of Acute Pancreatitis
}

\author{
Yingying Sun', Jie Jin $\mathbb{D}^{2}$, Aying Zhu', Hong Hu', Yingying Lu', Yue Zeng', Dadao Jing' \\ 'Department of Gastroenterology, Shanghai General Hospital, Shanghai Jiao Tong University School of Medicine, Shanghai, 20I600, People's Republic \\ of China; ${ }^{2}$ Department of Geriatrics, Shanghai General Hospital, Shanghai Jiao Tong University School of Medicine, Shanghai, 200080, People's \\ Republic of China
}

Correspondence: Dadao Jing, Department of Gastroenterology, Shanghai General Hospital, Shanghai Jiao Tong University School of Medicine, NO. 650 Xinsongjiang Road, Shanghai, 201600, People's Republic of China, Tel +86-I38I6958050, Email dadaojing@I26.com

Background: In recent years, the cure rate of acute pancreatitis (AP) has increased gradually, but the morbidity of recurrent acute pancreatitis (RAP) has not decreased. Patients with RAP have a poor quality of life and are more likely to develop into chronic pancreatitis. To investigate the risk factors of RAP after first pancreatitis attack is very necessary.

Methods: Patients with first episode AP admitted to Shanghai General Hospital, Shanghai Jiao Tong University School of Medicine from January 2018 to May 2021 were included in this retrospective study, and follow-up was 3-45 months after discharge. The patients information were collected from medical records including laboratory tests and auxiliary inspection of their hospitalization. Univariate and multivariate Cox regression analysis were used to explore the risk factors of RAP. Cumulative risk of RAP was plotted using Kaplan-Meier curves.

Results: A total of 592 patients were enrolled in the study and 81 (13.7\%) of the patients developed RAP. Among those RAP patients, the majority $(67.9 \%)$ were men, with a median age of 43 years. The most common etiology of RAP was hypertriglyceridemia (38.3\%). Multivariate Cox analysis showed that smoking history $(p<0.001)$, infected pancreatic necrosis occurred during first admission $(p=$ 0.005), and high low-density lipoprotein cholesterol (LDLc) level $(p<0.001)$ were significant independent risk factors for RAP. Patients with the above independent risk factors had increased 3-year cumulative risk of recurrence $(32.2 \%, 45.5 \%, 28.9 \%$, respectively).

Conclusion: Smoking history, infected pancreatic necrosis, and high LDLc level were the most decisive risk factors for RAP. Attention should be paid to the patients with the above factors.

Keywords: first episode pancreatitis, recurrent acute pancreatitis, risk factors, low-density lipoprotein cholesterol, infected pancreatic necrosis

\section{Introduction}

Acute pancreatitis (AP) is one of the most common critical diseases of the gastrointestinal tract. It is estimated that between 5 and 35 people per 100,000 are treated for AP annually, and this number has increased over the past decade. ${ }^{1}$ Biliary, hypertriglyceridemia and alcohol are the common causes of AP. Etiological composition varies in different countries and regions. ${ }^{2,3}$ Most AP can be completely recovered after standard treatment, but a few patients may have recurrent episodes. The recurrence rate of AP reported in previous studies is around $10-30 \%{ }^{2,4-6}$ It is important to note that patients with recurrent acute pancreatitis (RAP) are at high risk of progressing to chronic pancreatitis and even may be related to pancreatic cancer. ${ }^{7}$ And even in the absence of chronic pancreatitis, patients with RAP have lower physical and mental quality of life. ${ }^{8}$

It is necessary to find the risk factors of pancreatitis recurrence accurately and early. Previous studies have shown that factors that can lead to AP are more likely to become causes of RAP. ${ }^{9}$ Continued drinking after the first attack, delayed cholecystectomy, and poor control of triglyceride level are all well-known risk factors for RAP. ${ }^{9,10}$ The correlation between smoking and recurrence of AP is also clear, and it has been agreed that smokers have a significantly increased 
risk of recurrence. ${ }^{11-13}$ The purpose of this study was to find the risk factors for recurrence of AP by analyzing the laboratory test indicators and clinical characteristics of patients with the first episode AP.

\section{Materials and Methods}

\section{Patients and Study Design}

Patients admitted to Shanghai General Hospital, Shanghai Jiao Tong University School of Medicine with a first episode of AP between January 2018 and May 2021 were included in our study. The exclusion criteria included patients younger than 18 years of age, pregnancy, prior malignancy, chronic pancreatitis, and poor compliance. The inclusion and exclusion criteria of this study are shown in Figure 1. Patient characteristics, past medical history, tobacco and alcohol consumption, local complication, etiology and severity of AP, laboratory data and modified computed tomography severity index (MCTSI) were collected from medical records of their first hospitalization. All patients received standardized management according to guidelines and consensus. ${ }^{14}$ Follow-up of each patient began on the day of discharge and ended with a readmission for AP or the earliest of the following dates: death, loss of contact, or time to end date of our study: August 2021. The follow-up evaluation was based on medical records and telephone visit. This study

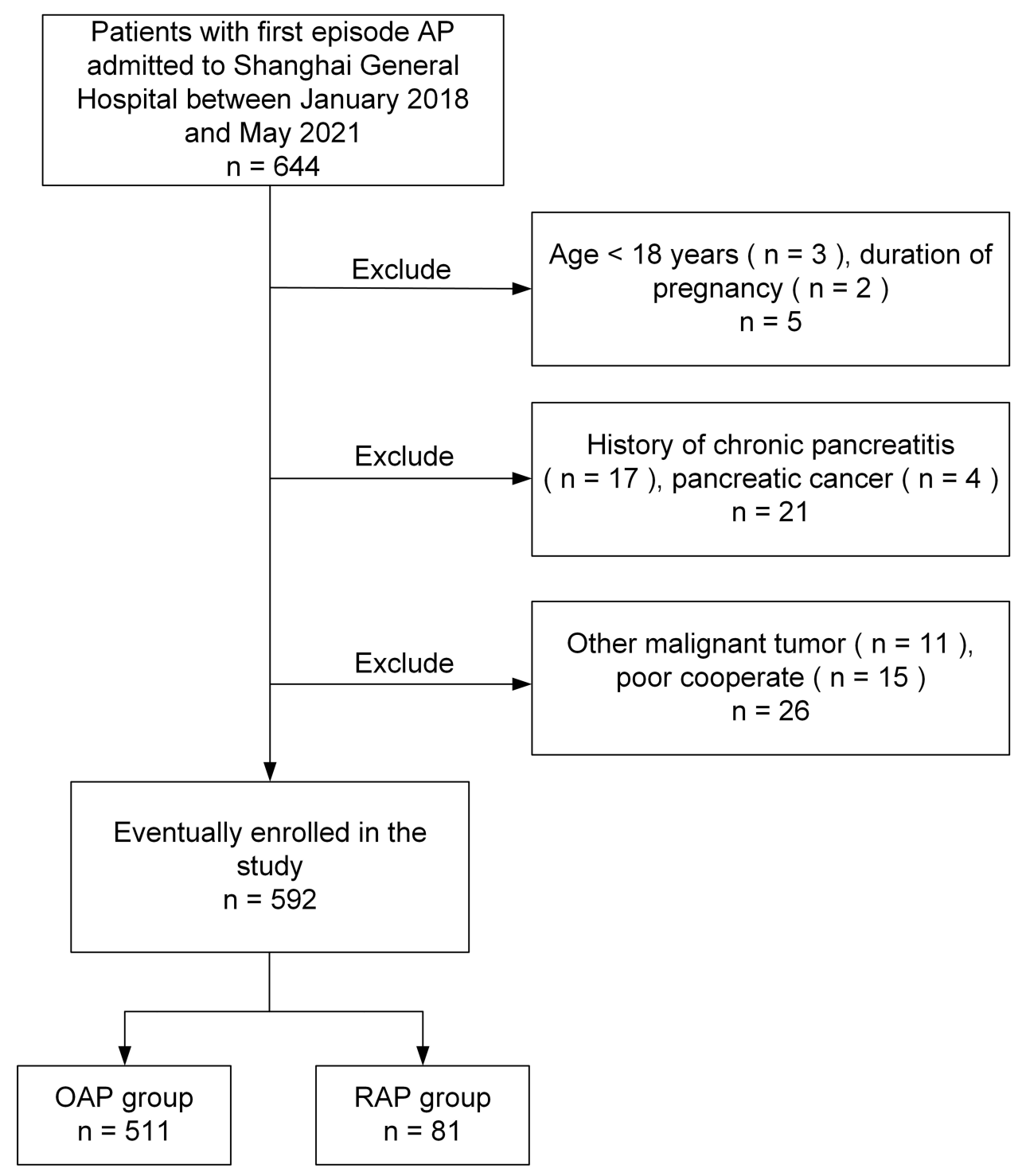

Figure I Flowchart of the patients included in the study.

Abbreviations: AP, acute pancreatitis; OAP, once episode of acute pancreatitis; RAP, recurrent acute pancreatitis. 
was reviewed and approved by the Medical Ethical Committee of Shanghai General Hospital, Shanghai Jiao Tong University School of Medicine (No. 021KY049).

\section{Definition}

The diagnosis and classification of AP were based on the 2012 revised Atlanta classification criteria. ${ }^{15}$ The diagnosis of AP required two of the following three criteria: 1) typical abdominal pain consistent with AP; 2) serum amylase (or lipase) activity at least three times greater than the standard upper limit; and 3) characteristic findings consistent with AP on computed tomography or abdominal ultrasonography. RAP was defined as follows: 1) interval between attacks greater than 3 months; 2) first episode AP entirely or near wholly resolved; and 3) no conclusive evidence of underlying chronic pancreatitis. ${ }^{16,17}$

Acute biliary pancreatitis (ABP) was defined as AP caused by biliary stones or biliary tract obstruction. ${ }^{15}$ Hypertriglyceridemic pancreatitis (HTGP) was described as a serum triglyceride level at admission greater than 11.3 $\mathrm{mmol} / \mathrm{L}$ or greater than $5.65 \mathrm{mmol} / \mathrm{L}$ without other causes, such as biliary stones or obstruction, and alcohol excluded. ${ }^{10}$ Alcohol-induced AP was diagnosed as the consumption of more than $80 \mathrm{~g}$ of alcohol per day for at least 10 years or heavy alcohol consumption prior to onset of AP. ${ }^{18-22}$ The remaining cases, such as autoimmune pancreatitis, idiopathic pancreatitis, pancreas divisum, intraductal papillary mucinous neoplasm were defined as others etiology of AP.

\section{Laboratory Detection}

Whole blood and serum samples were collected at the first time of admission. Whole blood samples were used to detect C-reactive protein and hemoglobin (BC-1050, Mindray, Guangzhou, China). Serum samples were used to detect the concentrations of blood calcium, fasting blood glucose, glycated hemoglobin A1c, albumin, total cholesterol, triglyceride, high-density lipoprotein cholesterol, low-density lipoprotein cholesterol (LDLc) (ADIVA2400, Siemens, Munich, Germany) and D-dimer (CN6000, Sysmex, Kobe, Japan). Their concentrations were obtained by standard operating methods.

All laboratory indicators were classified according to the normal reference range of our hospital. D-dimer level was classified as high or normal based on whether they were above the upper limit of normal $(0.55 \mathrm{mg} / \mathrm{L})$. Glycated hemoglobin A1c was divided into high and normal groups based on whether it was greater than $6 \%$. Triglyceride level higher than $1.7 \mathrm{mmol} / \mathrm{L}$ was classified as high-level. LDLc level was divided into the high-level group and the normal or low-level group according to whether they were greater than $4.14 \mathrm{mmol} / \mathrm{L}$. Serum calcium was divided into low $(<2.10 \mathrm{mmol} / \mathrm{L})$ and normal or high $(\geq 2.10 \mathrm{mmol} / \mathrm{L})$ groups. Fasting blood glucose was divided into high $(>6.11 \mathrm{mmol} / \mathrm{L})$ and normal or low $(\leq 6.11 \mathrm{mmol} / \mathrm{L})$ groups. Albumin was divided into low $(<40 \mathrm{~g} / \mathrm{L})$ and normal $(\geq 40 \mathrm{~g} / \mathrm{L})$ groups. Hemoglobin was divided into low $(<131 \mathrm{~g} / \mathrm{L})$ and normal or high $(\geq 131 \mathrm{~g} / \mathrm{L})$ groups. Total cholesterol was divided into high $(>5.18 \mathrm{mmol} / \mathrm{L})$ and normal $(\leq 5.18 \mathrm{mmol} / \mathrm{L})$ groups. High-density lipoprotein cholesterol was divided into low $(<1.16 \mathrm{mmol} / \mathrm{L})$ and normal or high $(\geq 1.16 \mathrm{mmol} / \mathrm{L})$ groups. C-reactive protein was divided into high and normal groups based on whether it was greater than $10 \mathrm{mg} / \mathrm{L}$.

\section{Statistical Analysis}

All statistical tests were conducted using SPSS version 26.0 and GraphPad Prism version 8.0. We expressed continuous variables as the mean \pm standard deviation $(\mathrm{Ma} \pm \mathrm{SD}$ ) or as the median \pm interquartile range (Md $\pm \mathrm{IQR})$. Normally distributed data were analyzed by a 2-tailed $t$-test, whereas non-normally distributed data tested by the Mann-Whitney $U$-test. Categorical variables were indicated as proportions and analyzed using the $\chi^{2}$ test. If more than $20 \%$ of the expected value was less than 5, Fisher's exact test was used. Variables with a $p<0.05$ on univariate analysis were entered into a multivariable Cox regression to identify independent predictors. Results were presented as hazard ratios (HRs) with 95\% confidence intervals (CIs). Cumulative incidences of RAP were plotted using Kaplan-Meier curves. Statistical significance was defined as $p<0.05$.

\section{Results}

\section{Patient Characteristics}

A total of 644 patients with first episode AP were admitted to the Shanghai General Hospital, Shanghai Jiao Tong University School of Medicine from January 2018 to May 2021. Data were excluded from the exclusion criteria (Age 
$<18$ years, pregnancy, chronic pancreatitis, pancreatic cancer, other malignant tumor and poor cooperate), and a total of 52 people were excluded. Eventually, 592 patients were enrolled in this study. According to whether patients have recurrence, we divided them into RAP group $(\mathrm{n}=81)$ and once episode of AP (OAP) group $(\mathrm{n}=511)$. Baseline characteristics are presented in Table 1 . The median age was $49 \pm 26$ years, median body mass index (BMI) was 25.40 $\pm 4.60 \mathrm{~kg} / \mathrm{m}^{2}$, and $383(64.7 \%)$ were males. Seventy-two (12.2\%) had a history of diabetes mellitus and $236(39.9 \%)$ had a previous diagnosis of fatty liver. The etiology of AP was biliary in 289 patients $(48.8 \%)$, hypertriglyceridemia (HTG) in 157 patients (26.5\%), alcohol in 68 patients (11.5\%), and others in 78 patients $(13.2 \%)$. In total, 133 (22.5\%) people were exposed to cigarette smoke and $150(25.3 \%)$ people have a history of drinking. Of all local complications, acute peripancreatic fluid collection was the most common occurring in 257 (43.4\%) patients during the first hospitalization.

\section{Comparison of RAP and OAP}

The patients were followed up for 3-45 months with a median follow-up time of 25 months. And, 13.7\% (81/592) of patients with first episode AP developed RAP. The median age of the RAP patients was $43 \pm 29.5$ years, which was younger than that of OAP patients ( $50 \pm 26$ years, $p=0.123)$. The proportion of males was higher in the RAP group (55/ $81,67.9 \%)$ than in the OAP group $(328 / 511,64.2 \%)$, but the difference was not statistically significant $(p=0.516)$. The median BMI for two groups were $25.8 \mathrm{~kg} / \mathrm{m}^{2}$ and $25.4 \mathrm{~kg} / \mathrm{m}^{2}$, respectively $(p=0.459)$. There were significant differences in tobacco smoke exposure $(p<0.001)$ and alcohol consumption $(p<0.001)$ between the two groups. There was no significant difference in previous diagnosis of diabetes mellitus $(p=0.674)$ between the two groups while the RAP group had a higher rate of fatty liver history $(p=0.004)$.

The composition of etiology of AP was significantly different $(p=0.010)$. The most common etiology in the OAP group was biliary (51.5\%), whereas the etiology was HTG (38.3\%) in the RAP group. Of the 12 patients (14.8\%) with others etiology in RAP group, all patients had completed endoscopic ultrasonography during the follow-up. Six of them were congenital malformation of the pancreas (pancreas divisum), four were idiopathic pancreatitis, and the rest of them were autoimmune pancreatitis and intraductal papillary mucinous neoplasm.

The incidence of acute peripancreatic fluid collection, pancreatic pseudocyst, and infected pancreatic necrosis in RAP group were significantly higher than that in OAP group during the first hospitalization $(p=0.000, p=0.021, p=0.010$, respectively). There were significant differences in serum calcium, fasting blood glucose, and LDLc level between the two groups ( $p=0.040, p=0.015, p=0.000$, respectively). Differences in clinical feature profiles between RAP and OAP groups are presented in Table 1.

\section{Risk of RAP}

Regression analysis of risk factors for RAP is presented in Table 2. After univariate Cox regression analysis, the cigarette smoke exposure, alcohol consumption, fatty liver history, etiology and severity of AP, MCTSI, acute peripancreatic fluid collection, pancreatic pseudocyst, infected pancreatic necrosis, high fasting blood glucose level, high triglyceride level, and high LDLc level were significantly associated with RAP $(p<0.05)$. There is no correlation between the respective variables. Multivariate Cox regression analysis of these variables showed that cigarette smoke exposure $(p<0.001)$, infected pancreatic necrosis $(p=0.005)$ and high LDLc level $(p<0.001)$ were significantly independent risk factors for RAP.

Cumulative risk analysis of development of RAP stratified by cigarette smoke exposure, infected pancreatic necrosis and LDLc level is presented in Figure 2. The 3-year cumulative risk for RAP was highest in patients with infected pancreatic necrosis during the first episode pancreatitis (45.5\%). Smokers or patients with high LDLc level have significantly higher cumulative risk for RAP over 3 years than non-smokers or patients with normal LDLc level (32.2\% vs $9.5 \%, 28.9 \%$ vs $5 \%$, respectively).

\section{Discussion}

Recurrent episodes of pancreatitis seriously affect the quality of life. ${ }^{8}$ In addition, some studies consider that AP, RAP, and chronic pancreatitis are the continuum of the same disease. ${ }^{23}$ It has even been suggested that RAP may be an 
Table I Basic Characteristics of Patients with First Episode Acute Pancreatitis

\begin{tabular}{|c|c|c|c|c|}
\hline & $\begin{array}{l}\text { All Patients } \\
(n=592)\end{array}$ & $\begin{array}{c}\text { OAP } \\
(n=5 I I)\end{array}$ & $\begin{array}{c}\text { RAP } \\
(n=81)\end{array}$ & $p$ \\
\hline \multicolumn{5}{|l|}{ Sex } \\
\hline Male & $383(64.7 \%)$ & $328(64.2 \%)$ & 55 (67.9\%) & $0.516^{\mathrm{a}}$ \\
\hline Female & 209 (35.3\%) & I83 (35.8\%) & $26(32.1 \%)$ & \\
\hline Age (years) & $49.0 \pm 26.0$ & $50.0 \pm 26.0$ & $43.0 \pm 29.5$ & $0.123^{b}$ \\
\hline BMI $\left(\mathrm{kg} / \mathrm{m}^{2}\right)$ & $25.40 \pm 4.60$ & $25.40 \pm 4.47$ & $25.80 \pm 4.95$ & $0.459^{b}$ \\
\hline \multicolumn{5}{|l|}{ Habits } \\
\hline Smoking & I 33 (22.5\%) & $93(18.2 \%)$ & 40 (49.4\%) & $<0.00 I^{\mathrm{a}}$ \\
\hline Drinking & $150(25.3 \%)$ & 116 (22.7\%) & $34(42.0 \%)$ & $<0.001^{\mathrm{a}}$ \\
\hline Diabetes mellitus & $72(12.2 \%)$ & 61 (11.9\%) & II (I3.6\%) & $0.674^{\mathrm{a}}$ \\
\hline Fatty liver & 236 (39.9\%) & $192(37.6 \%)$ & $44(54.3 \%)$ & $0.004^{\mathrm{a}}$ \\
\hline \multicolumn{5}{|l|}{ Etiology of AP } \\
\hline Biliary & 289 (48.8\%) & $263(51.5 \%)$ & $26(32.1 \%)$ & $0.010^{\mathrm{a}}$ \\
\hline HTG & 157 (26.5\%) & $126(24.7 \%)$ & $31(38.3 \%)$ & \\
\hline Alcohol & 68 (11.5\%) & 56 (II.0\%) & $12(14.8 \%)$ & \\
\hline Others & 78 (I3.2\%) & 66 (12.9\%) & 12 (I4.8\%) & \\
\hline \multicolumn{5}{|l|}{ Local complication } \\
\hline Acute peripancreatic fluid collection & 257 (43.4\%) & $206(40.3 \%)$ & $5 \mathrm{I}(63.0 \%)$ & $<0.001^{\mathrm{a}}$ \\
\hline Acute necrotic collection & $58(9.8 \%)$ & $48(9.4 \%)$ & $10(12.3 \%)$ & $0.406^{\mathrm{a}}$ \\
\hline Pancreatic pseudocyst & $27(4.6 \%)$ & $19(3.7 \%)$ & $8(9.9 \%)$ & $0.021^{\mathrm{a}}$ \\
\hline Walled-off necrosis & $14(2.4 \%)$ & $10(2.0 \%)$ & $4(4.9 \%)$ & $0.111^{\mathrm{a}}$ \\
\hline Infected pancreatic necrosis & $11(1.9 \%)$ & $6(1.2 \%)$ & $5(6.2 \%)$ & $0.010^{\mathrm{a}}$ \\
\hline \multicolumn{5}{|l|}{ MCTSI } \\
\hline Mild & $212(35.8 \%)$ & $194(38.0 \%)$ & $18(22.2 \%)$ & $0.023^{\mathrm{a}}$ \\
\hline Moderate & $328(55.4 \%)$ & $274(53.6 \%)$ & $54(66.7 \%)$ & \\
\hline Severe & $52(8.8 \%)$ & $43(8.4 \%)$ & $9(11.1 \%)$ & \\
\hline \multicolumn{5}{|l|}{ Severity of AP } \\
\hline MAP & $368(62.2 \%)$ & $325(63.6 \%)$ & $43(53.1 \%)$ & $0.107^{\mathrm{a}}$ \\
\hline MSAP & $|5|(25.5 \%)$ & $128(25.0 \%)$ & $23(28.4 \%)$ & \\
\hline SAP & $73(12.3 \%)$ & 58 (II.4\%) & 15 (I8.5\%) & \\
\hline \multicolumn{5}{|l|}{ Laboratory test } \\
\hline High D-dimer & 466 (78.7\%) & 403 (78.9\%) & $63(77.8 \%)$ & $0.824^{\mathrm{a}}$ \\
\hline Low-Ca ${ }^{2+}$ & 231 (39.0\%) & 191 (37.4\%) & 40 (49.4\%) & $0.040^{\mathrm{a}}$ \\
\hline High-FBG & 343 (57.9\%) & $286(56.0 \%)$ & 57 (70.4\%) & $0.015^{\mathrm{a}}$ \\
\hline High-HbAlc & $188(31.8 \%)$ & 154 (30.1\%) & $34(42.0 \%)$ & $0.033^{\mathrm{a}}$ \\
\hline Low-ALB & 314 (53.0\%) & 267 (52.3\%) & 47 (58.0\%) & $0.333^{\mathrm{a}}$ \\
\hline Low-Hb & $184(31.1 \%)$ & 159 (31.1\%) & 25 (30.9\%) & $0.964^{a}$ \\
\hline High-TC & 229 (38.7\%) & 192 (37.6\%) & 37 (45.7\%) & $0.164^{\mathrm{a}}$ \\
\hline High-TG & 234 (39.5\%) & 190 (37.2\%) & $44(54.3 \%)$ & $0.003^{\mathrm{a}}$ \\
\hline Low-HDLc & 434 (73.3\%) & 370 (72.4\%) & 64 (79.0\%) & $0.212^{\mathrm{a}}$ \\
\hline High-LDLc & 64 (10.8\%) & 32 (6.3\%) & 32 (39.5\%) & $<0.001^{\circ}$ \\
\hline High-CRP & 477 (80.6\%) & 409 (80.0\%) & $68(84.0 \%)$ & $0.408^{\mathrm{a}}$ \\
\hline
\end{tabular}

Notes: ${ }^{a} \chi^{2}$ test, categorical variables were indicated as proportions. ${ }^{b}$ Mann-Whitney $U$-test, continuous variables as the median with the interquartile range. Abbreviations: OAP, once episode of acute pancreatitis; RAP, recurrent acute pancreatitis; BMI, body mass index; HTG, hypertriglyceridemia; MCTSI, modified computed tomography severity index; MAP, mild acute pancreatitis; MSAP, moderately severe acute pancreatitis; SAP, severe acute pancreatitis; $\mathrm{Ca}^{2+}$, serum calcium; FBG, fasting blood glucose; HbAlc, glycated hemoglobin Alc; ALB, albumin; Hb, hemoglobin; TC, total cholesterol; TG, triglyceride; HDLc, high-density lipoprotein cholesterol; LDLc, low-density lipoprotein cholesterol; CRP, C-reactive protein.

independent risk factor for pancreatic cancer. ${ }^{7}$ The occurrence of RAP was found to be $13.7 \%$ during follow-up, which was consistent with the conclusion of other studies. ${ }^{2}$

The etiology composition of AP varies between countries and regions. ${ }^{2,3,24}$ It has been reported that HTG has become the second most common cause after biliary in China. ${ }^{2,24}$ In our study, HTG and alcohol etiology caused more 
Table 2 Cox Regression Analyses of the Risk Factors Associated with Recurrent Acute Pancreatitis

\begin{tabular}{|c|c|c|c|c|}
\hline & $\begin{array}{c}\text { Univariate Analysis } \\
\text { HR }(95 \% \mathrm{Cl})\end{array}$ & $p$ & $\begin{array}{c}\text { Multivariate Analysis } \\
\text { HR }(95 \% \mathrm{Cl})\end{array}$ & $p$ \\
\hline \multicolumn{5}{|l|}{ Sex } \\
\hline Male & $0.883(0.554-1.408)$ & 0.600 & & \\
\hline Female & & & & \\
\hline Age (years) & $0.990(0.977-1.004)$ & 0.171 & & \\
\hline BMI $\left(\mathrm{kg} / \mathrm{m}^{2}\right)$ & $1.014(0.958-1.074)$ & 0.631 & & \\
\hline \multicolumn{5}{|l|}{ Habits } \\
\hline Smoking & $3.621(2.342-5.598)$ & $<0.001$ & $3.512(2.010-6.139)$ & $<0.001$ \\
\hline Drinking & $2.178(1.40 \mathrm{I}-3.387)$ & 0.001 & $1.326(0.728-2.412)$ & 0.356 \\
\hline Diabetes mellitus & $1.164(0.616-2.197)$ & 0.641 & & \\
\hline Fatty liver & $1.825(1.179-2.826)$ & 0.007 & $1.026(0.593-1.775)$ & 0.926 \\
\hline \multicolumn{5}{|l|}{ Etiology of AP } \\
\hline Biliary & $\operatorname{Ref}(1.00)$ & & $\operatorname{Ref}(1.00)$ & \\
\hline HTG & $2.201(1.307-3.707)$ & 0.003 & $1.078(0.488-2.378)$ & 0.853 \\
\hline Alcohol & $1.998(1.008-3.959)$ & 0.047 & $0.67 \mid(0.283-1.591)$ & 0.364 \\
\hline Others & $1.723(0.869-3.414)$ & 0.119 & $1.709(0.812-3.595)$ & 0.158 \\
\hline \multicolumn{5}{|l|}{ Local complication } \\
\hline $\begin{array}{l}\text { Acute peripancreatic fluid } \\
\text { collection }\end{array}$ & $2.243(1.429-3.521)$ & $<0.001$ & $1.608(0.760-3.40 \mathrm{I})$ & 0.214 \\
\hline Acute necrotic collection & $1.372(0.707-2.659)$ & 0.350 & & \\
\hline Pancreatic pseudocyst & $2.628(1.266-5.455)$ & 0.010 & $1.952(0.818-4.659)$ & 0.132 \\
\hline Walled-off necrosis & $2.460(0.900-6.723)$ & 0.079 & & \\
\hline Infected pancreatic necrosis & $4.908(1.982-12.151)$ & 0.001 & $5.014(1.643-15.294)$ & 0.005 \\
\hline \multicolumn{5}{|l|}{ MCTSI } \\
\hline Mild & $\operatorname{Ref}(1.00)$ & & $\operatorname{Ref}(1.00)$ & \\
\hline Moderate & 1.957 (I.148-3.337) & 0.014 & $1.209(0.567-2.578)$ & 0.624 \\
\hline Severe & $2.169(0.974-4.829)$ & 0.058 & $0.72 I(0.2 I I-2.46 I)$ & 0.602 \\
\hline \multicolumn{5}{|l|}{ Severity of AP } \\
\hline MAP & $\operatorname{Ref}(1.00)$ & & $\operatorname{Ref}(1.00)$ & \\
\hline MSAP & $1.272(0.767-2.1 \mathrm{II})$ & 0.351 & $0.647(0.348-1.204)$ & 0.169 \\
\hline SAP & $1.885(1.047-3.393)$ & 0.035 & I.I0I (0.488-2.483) & 0.817 \\
\hline \multicolumn{5}{|l|}{ Laboratory test } \\
\hline High D-dimer & $0.933(0.553-1.576)$ & 0.797 & & \\
\hline Low-Ca ${ }^{2+}$ & $1.262(0.783-2.034)$ & 0.339 & & \\
\hline High-FBG & $1.33 \mid(1.048-1.689)$ & 0.019 & $1.356(0.785-2.342)$ & 0.274 \\
\hline High-HbAlc & $1.553(0.999-2.416)$ & 0.051 & & \\
\hline Low-ALB & $1.148(0.738-1.787)$ & 0.540 & & \\
\hline Low-Hb & $0.99 \mid(0.618-1.588)$ & 0.970 & & \\
\hline High-TC & $1.367(0.883-2.118)$ & 0.161 & & \\
\hline High-TG & $1.842(1.189-2.851)$ & 0.006 & $0.905(0.445-1.842)$ & 0.783 \\
\hline Low-HDLc & $1.382(0.810-2.360)$ & 0.236 & & \\
\hline High-LDLc & $2.548(2.038-3.186)$ & $<0.001$ & $5.036(3.067-8.267)$ & $<0.001$ \\
\hline High-CRP & $1.251(0.691-2.264)$ & 0.460 & & \\
\hline
\end{tabular}

Note: Risk factors showing potential association $(p<0.05)$ in univariate analysis were subsequently entered into a multivariable regression.

Abbreviations: BMI, body mass index; HTG, hypertriglyceridemia; MCTSI, modified computed tomography severity index; MAP, mild acute pancreatitis; MSAP, moderately severe acute pancreatitis; SAP, severe acute pancreatitis; $\mathrm{Ca}^{2+}$, serum calcium; FBG, fasting blood glucose; HbAIc, glycated hemoglobin AIc; ALB, albumin; Hb, hemoglobin; TC, total cholesterol; TG, triglyceride; HDLc, high-density lipoprotein cholesterol; LDLc, low-density lipoprotein cholesterol; CRP, C-reactive protein.

AP recurrence than biliary, and HTG is the most frequent etiology factor of recurrence. This result may be related to poor lipid control and continued alcohol consumption after discharge. Tai et al also found higher recurrence rate of HTGP than ABP. ${ }^{25}$ HTGP is often associated with multiple factors (metabolic abnormalities, lack of exercise, alcohol 


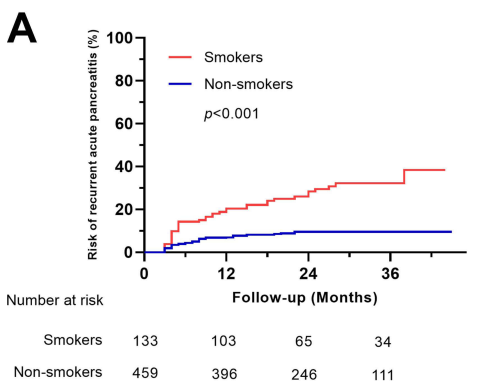

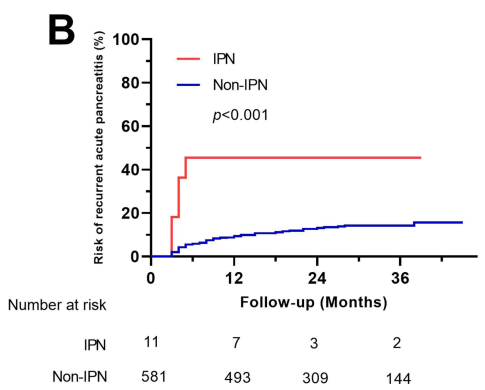

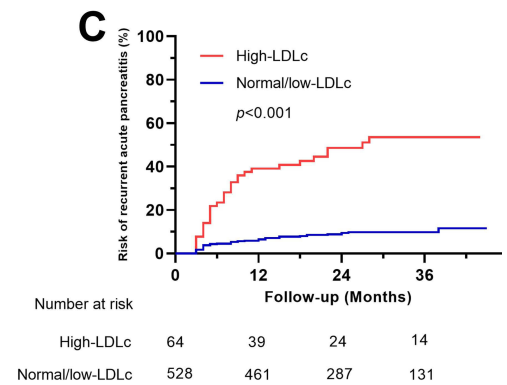

Figure 2 Cumulative risk for recurrent acute pancreatitis after first episode acute pancreatitis. (A) For recurrent acute pancreatitis based on tobacco consumption; (B) for recurrent acute pancreatitis based on IPN; (C) for recurrent acute pancreatitis based on LDLc levels.

Abbreviations: IPN, infected pancreatic necrosis; LDLc, low-density lipoprotein cholesterol.

intake, microcirculation disorders) and is more severe and has a worse prognosis than other causes, which may also contribute to its higher recurrence rate. We also found a proportion of patients with pancreas congenital malformation in the RAP group. Earlier studies had shown that pancreas divisum is associated with RAP, which may be related to sustained ductal hypertension due to narrower minor papilla and minor duct. ${ }^{26,27}$ Bertin et al suggested that pancreas divisum itself was not a cause of pancreatitis and that pancreatitis occurred as a cumulative effect of CFTR mutations and pancreas divisum. ${ }^{28}$ The different etiologies of acute pancreatitis and the mechanisms associated with the recurrence of acute pancreatitis remain to be further explored. As is known to all that factors that can lead to AP are more likely to become causes of RAP. ${ }^{9,29}$ It is essential to determine the etiology of the disease in time to apply the most appropriate therapy.

There seems to be a consensus that cigarette consumption is a risk factor for RAP. ${ }^{5,11-13}$ We found that smokers had a significantly higher cumulative risk for RAP than non-smokers. However, the mechanism by which cigarette consumption increases the recurrence rate of pancreatitis remains unclear. Wittel et al suggested that tobacco smoke exposure causes pancreatic damage, in part, by altering the balance between trypsinogen and pancreas-specific trypsin inhibitor, which creates a microenvironment that is conducive to pancreatic autodigestion and promotes acinar cell injury. ${ }^{30}$ Chowdhury et al showed that nicotine can activate multiple pathways in acinar cells and induce oxidative stress. ${ }^{31}$ These changes cause calcium influx leading to cell injury. In addition, we suspect that chronic vascular damage caused by smoking may also be a factor of recurrence. Previous studies have shown that exposure to tobacco smoke impairs the function of vascular endothelial cells, but most of the research focused on coronary arteries. ${ }^{32-34}$ Further research is needed on the pathogenesis of RAP caused by smoking and whether smoking cessation can reduce the risk of recurrence. Anyway, physicians should be advising patients to quit smoking at all times.

In this study, we found that high LDLc level is associated with pancreatitis recurrence. As far as we know, this is the first time that the conclusion has been put forward. It is well known that high LDLc level triggers a vicious cycle of oxidative stress that leads to vascular endothelial dysfunction. ${ }^{35}$ We suspect this may be related to recurrent episodes of pancreatitis. We know that one consequence of pancreatitis is the vascular damage within the pancreas. ${ }^{36,37}$ However, whether chronic vascular endothelial dysfunction leads to pancreatic vulnerable is unknown. Due to the effects of cigarette smoke exposure and high LDLc level on vascular injury are clear, we hypothesized that these two factors may contribute to recurrence of pancreatitis by causing chronic vascular damage. ${ }^{32,33,35,38}$

The link between RAP and pancreatic cancer was not fully elaborated. ${ }^{38}$ A population-based cohort study found that RAP may be an independent risk factor for pancreatic cancer. ${ }^{7}$ Although RAP can be a sign of pancreatic cancer, it is also plausible that inflammatory process of the pancreas triggers the development of pancreatic cancer. Identifying the risk factors for recurrence of AP can not only prevent RAP, but also reduce the incidence of pancreatic cancer possibly. Previous studies have shown that the risk of AP in diabetic patients is 1.86-2.89 times higher than that in the general population. ${ }^{22,39-41}$ However, there are few studies on whether first episode AP with diabetes mellitus increases the risk of pancreatitis recurrence. Our research found that patients with high-HbA1c at 
admission for the first attack of AP were more likely to have RAP. Unfortunately, this is not an independent risk factor for recurrence of AP.

The severity of the first episode of AP does not seem to be significantly related to the recurrence in this study. This is consistent with previous research. ${ }^{22,41}$ Cho et al found that a local complication at index admission in patients with first episode pancreatitis was the strongest risk factor for RAP. ${ }^{42}$ The occurrence of local complications may be related to structural changes in the pancreas after recovery from pancreatitis. Our regression analysis found that patients who developed infected pancreatic necrosis during their first hospitalization had a fivefold higher risk of recurrence than those who without. A cross-sectional study in the Netherlands found that pancreatic necrosis was an independent risk factor for recurrent pancreatitis. ${ }^{11}$ The scar formed during the healing process of pancreatic necrosis, especially the scar of pancreatic duct, may be an important cause of pancreatitis repeated attacks.

There are a number of limitations in our study. First, this is a observation study conducted in a single center. Second, our cases were followed for a short time that the incidence of RAP may be underestimated. A longer follow-up duration may have provided further insights. Third, the effect of smoking on recurrence of pancreatitis was not quantified. Fourth, our strict definition of alcohol-induced AP may understate the incidence of this etiology. In the future, we will verify our results in pathophysiology and design large-scale prospective studies to quantify risk factors.

\section{Conclusions}

In this study, $13.7 \%$ of the patients developed RAP after a first-attack of AP. Smokers, patients with infected pancreatic necrosis during first admission and patients with high LDLc level had significantly higher cumulative risk for RAP over 3 years. Cigarette consumption, infected pancreatic necrosis and high LDLc level were independent risk factors of RAP. In the future, we should help patients improve their awareness of risk factors for RAP, improve compliance behavior and advocate a healthy lifestyle, so as to prevent recurrence of pancreatitis.

\section{Abbreviations}

OAP, once episode of acute pancreatitis; RAP, recurrent acute pancreatitis; BMI, body mass index; HTG, hypertriglyceridemia; MCTSI, modified computed tomography severity index; MAP, mild acute pancreatitis; MSAP, moderately severe acute pancreatitis; SAP, severe acute pancreatitis; $\mathrm{Ca}^{2+}$, serum calcium; FBG, fasting blood glucose; $\mathrm{HbA1c}$, glycated hemoglobin A1c; ALB, albumin; Hb, hemoglobin; TC, total cholesterol; TG, triglyceride; HDLc, high-density lipoprotein cholesterol; LDLc, low-density lipoprotein cholesterol; CRP, C-reactive protein; IPN, infected pancreatic necrosis.

\section{Data Sharing Statement}

The data used and analyzed in the current study can be made available upon request to the corresponding author.

\section{Ethics Approval and Consent to Participate}

The study has been ethically approved by the ethics committee of Shanghai General Hospital, Shanghai Jiao Tong University School of Medicine (IRB No. 021KY049). The respondents were informed about the aim of the study. The respondents provided informed consent for the survey. This study was conducted in accordance with the declaration of Helsinki. The confidentiality and anonymity of the data was also ensured.

\section{Author Contributions}

All authors made a significant contribution to the work reported, whether that is in the conception, study design, execution, acquisition of data, analysis and interpretation, or in all these areas; took part in drafting, revising or critically reviewing the article; gave final approval of the version to be published; have agreed on the journal to which the article has been submitted; and agree to be accountable for all aspects of the work.

\section{Funding}

All costs involved in the study were covered by the authors with no additional funding being received. 


\section{Disclosure}

The authors report no conflicts of interest in this work.

\section{References}

1. Ingraham NE, King S, Proper J, et al. Morbidity and mortality trends of pancreatitis: an observational study. Surg Infect (Larchmt). 2021;22 (10):1021-1030. doi:10.1089/sur.2020.473

2. Guo YY, Li HX, Zhang Y, He WH. Hypertriglyceridemia-induced acute pancreatitis: progress on disease mechanisms and treatment modalities. Discov Med. 2019;27(147):101-109.

3. Pu W, Luo G, Chen T, et al. A 5-year retrospective cohort study: epidemiology, etiology, severity, and outcomes of acute pancreatitis. Pancreas. 2020;49(9):1161-1167. doi:10.1097/MPA.0000000000001637

4. Zafrir B, Saliba W, Jubran A, Hijazi R, Shapira C. Severe hypertriglyceridemia-related pancreatitis: characteristics and predictors of recurrence. Pancreas. 2019;48(2):182-186. doi:10.1097/MPA.0000000000001235

5. Magnusdottir BA, Baldursdottir MB, Kalaitzakis E, Bjornsson ES. Risk factors for chronic and recurrent pancreatitis after first attack of acute pancreatitis. Scand J Gastroenterol. 2019;54(1):87-94. doi:10.1080/00365521.2018.1550670

6. Stigliano S, Belisario F, Piciucchi M, Signoretti M, Delle FG, Capurso G. Recurrent biliary acute pancreatitis is frequent in a real-world setting. Dig Liver Dis. 2018;50(3):277-282. doi:10.1016/j.dld.2017.12.011

7. Sadr-Azodi O, Oskarsson V, Discacciati A, Videhult P, Askling J, Ekbom A. Pancreatic cancer following acute pancreatitis: a population-based matched cohort study. Am J Gastroenterol. 2018;113(11):1711-1719. doi:10.1038/s41395-018-0255-9

8. Cote GA, Yadav D, Abberbock JA, et al. Recurrent acute pancreatitis significantly reduces quality of life even in the absence of overt chronic pancreatitis. Am J Gastroenterol. 2018;113(6):906-912. doi:10.1038/s41395-018-0087-7

9. Yu B, Li J, Li N, et al. Progression to recurrent acute pancreatitis after a first attack of acute pancreatitis in adults. Pancreatology. 2020;20 (7):1340-1346. doi:10.1016/j.pan.2020.09.006

10. Wu BU, Batech M, Dong EY, Duan L, Yadav D, Chen W. Influence of ambulatory triglyceride levels on risk of recurrence in patients with hypertriglyceridemic pancreatitis. Dig Dis Sci. 2019;64(3):890-897. doi:10.1007/s10620-018-5226-X

11. Ahmed AU, Issa Y, Hagenaars JC, et al. Risk of recurrent pancreatitis and progression to chronic pancreatitis after a first episode of acute pancreatitis. Clin Gastroenterol Hepatol. 2016;14(5):738-746. doi:10.1016/j.cgh.2015.12.040

12. Munigala S, Conwell DL, Gelrud A, Agarwal B. Heavy smoking is associated with lower age at first episode of acute pancreatitis and a higher risk of recurrence. Pancreas. 2015;44(6):876-881. doi:10.1097/MPA.0000000000000364

13. Majumder S, Gierisch JM, Bastian LA. The association of smoking and acute pancreatitis: a systematic review and meta-analysis. Pancreas. 2015;44(4):540-546. doi:10.1097/MPA.0000000000000301

14. Mederos MA, Reber HA, Girgis MD. Acute pancreatitis: a review. JAMA. 2021;325(4):382-390. doi:10.1001/jama.2020.20317

15. Banks PA, Bollen TL, Dervenis C, et al. Classification of acute pancreatitis-2012: revision of the Atlanta classification and definitions by international consensus. Gut. 2013;62(1):102-111. doi:10.1136/gutjnl-2012-302779

16. Lloret LC, Pelletier AL, Czernichow S, et al. Acute pancreatitis in a cohort of 129 patients referred for severe hypertriglyceridemia. Pancreas. 2008;37(1):13-22. doi:10.1097/MPA.0b013e31816074a1

17. Gullo L, Migliori M, Olah A, et al. Acute pancreatitis in five European countries: etiology and mortality. Pancreas. 2002;24(3):223-227. doi:10.1097/00006676-200204000-00003

18. Gao YJ, Li YQ, Wang Q, et al. Analysis of the clinical features of recurrent acute pancreatitis in China. J Gastroenterol. 2006;41(7):681-685. doi:10.1007/s00535-006-1820-3

19. Dufour MC, Adamson MD. The epidemiology of alcohol-induced pancreatitis. Pancreas. 2003;27(4):286-290. doi:10.1097/00006676-20031100000002

20. Sand J, Lankisch PG, Nordback I. Alcohol consumption in patients with acute or chronic pancreatitis. Pancreatology. 2007;7(2-3):147-156. doi:10.1159/000104251

21. Argueta PP, Salazar M, Vohra I, et al. Thirty-day readmission among patients with alcoholic acute pancreatitis. Dig Dis Sci. 2021;66 (12):4227-4236. doi:10.1007/s10620-020-06765-7

22. Girman CJ, Kou TD, Cai B, et al. Patients with type 2 diabetes mellitus have higher risk for acute pancreatitis compared with those without diabetes. Diabetes Obes Metab. 2010;12(9):766-771. doi:10.1111/j.1463-1326.2010.01231.x

23. Sankaran SJ, Xiao AY, Wu LM, Windsor JA, Forsmark CE, Petrov MS. Frequency of progression from acute to chronic pancreatitis and risk factors: a meta-analysis. Gastroenterology. 2015;149(6):1490-1500.e1. doi:10.1053/j.gastro.2015.07.066

24. Berger Z, Mancilla C, Tobar E, et al. Acute pancreatitis in Chile: a multicenter study on epidemiology, etiology and clinical outcome. Retrospective analysis of clinical files. Pancreatology. 2020;20(4):637-643. doi:10.1016/j.pan.2020.04.016

25. Tai WP, Lin XC, Liu H, et al. A retrospective research of the characteristic of hypertriglyceridemic pancreatitis in Beijing, China. Gastroenterol Res Pract. 2016;2016:6263095. doi:10.1155/2016/6263095

26. Talukdar R, Aslam M, Reddy DN, et al. Pancreas divisum increases the risk of recurrent acute pancreatitis in patients with rs 12338 polymorphism in the Cathepsin B gene. Dig Dis Sci. 2021;66(7):2283-2290. doi:10.1007/s10620-020-06517-7

27. Fogel EL, Toth TG, Lehman GA, DiMagno MJ, DiMagno EP. Does endoscopic therapy favorably affect the outcome of patients who have recurrent acute pancreatitis and pancreas divisum? Pancreas. 2007;34(1):21-45. doi:10.1097/mpa.0b013e31802ce068

28. Bertin C, Pelletier AL, Vullierme MP, et al. Pancreas divisum is not a cause of pancreatitis by itself but acts as a partner of genetic mutations. Am J Gastroenterol. 2012;107(2):311-317. doi:10.1038/ajg.2011.424

29. Balint ER, Fur G, Kiss L, et al. Assessment of the course of acute pancreatitis in the light of aetiology: a systematic review and meta-analysis. Sci Rep. 2020;10(1):17936. doi:10.1038/s41598-020-74943-8

30. Wittel UA, Singh AP, Henley BJ, et al. Cigarette smoke-induced differential expression of the genes involved in exocrine function of the rat pancreas. Pancreas. 2006;33(4):364-370. doi:10.1097/01.mpa.0000240601.80570.31 
31. Chowdhury P, Walker A. A cell-based approach to study changes in the pancreas following nicotine exposure in an animal model of injury. Langenbecks Arch Surg. 2008;393(4):547-555. doi:10.1007/s00423-007-0267-1

32. Ma B, Chen Y, Wang X, et al. Cigarette smoke exposure impairs lipid metabolism by decreasing low-density lipoprotein receptor expression in hepatocytes. Lipids Health Dis. 2020;19(1):88. doi:10.1186/s12944-020-01276-w

33. Fu X, Zong T, Yang P, et al. Nicotine: regulatory roles and mechanisms in atherosclerosis progression. Food Chem Toxicol. $2021 ; 151: 112154$. doi:10.1016/j.fct.2021.112154

34. El-Mahdy MA, Abdelghany TM, Hemann C, et al. Chronic cigarette smoke exposure triggers a vicious cycle of leukocyte and endothelial-mediated oxidant stress that results in vascular dysfunction. Am J Physiol Heart Circ Physiol. 2020;319(1):H51-H65. doi:10.1152/ajpheart.00657.2019

35. Nakamura T, Uematsu M, Yoshizaki T, Kobayashi T, Watanabe Y, Kugiyama K. Improvement of endothelial dysfunction is mediated through reduction of remnant lipoprotein after statin therapy in patients with coronary artery disease. $J$ Cardiol. 2020;75(3):270-274. doi:10.1016/j. jjcc.2019.08.006

36. Afghani E, Pandol SJ, Shimosegawa T, et al. Acute pancreatitis-progress and challenges: a report on an international symposium. Pancreas. 2015;44(8):1195-1210. doi:10.1097/MPA.0000000000000500

37. Cuthbertson CM, Christophi C. Disturbances of the microcirculation in acute pancreatitis. Br J Surg. 2006;93(5):518-530. doi:10.1002/bjs.5316

38. Burke A, Fitzgerald GA. Oxidative stress and smoking-induced vascular injury. Prog Cardiovasc Dis. 2003;46(1):79-90. doi:10.1016/s00330620(03)00076-8

39. Urushihara H, Taketsuna M, Liu Y, et al. Increased risk of acute pancreatitis in patients with type 2 diabetes: an observational study using a Japanese hospital database. PLoS One. 2012;7(12):e53224. doi:10.1371/journal.pone.0053224

40. Lai SW, Muo CH, Liao KF, Sung FC, Chen PC. Risk of acute pancreatitis in type 2 diabetes and risk reduction on anti-diabetic drugs: a population-based cohort study in Taiwan. Am J Gastroenterol. 2011;106(9):1697-1704. doi:10.1038/ajg.2011.155

41. Noel RA, Braun DK, Patterson RE, Bloomgren GL. Increased risk of acute pancreatitis and biliary disease observed in patients with type 2 diabetes: a retrospective cohort study. Diabetes Care. 2009;32(5):834-838. doi:10.2337/dc08-1755

42. Cho JH, Jeong YH, Kim KH, Kim TN. Risk factors of recurrent pancreatitis after first acute pancreatitis attack: a retrospective cohort study. Scand $J$ Gastroenterol. 2020;55(1):90-94. doi:10.1080/00365521.2019.1699598

International Journal of General Medicine

\section{Publish your work in this journal}

The International Journal of General Medicine is an international, peer-reviewed open-access journal that focuses on general and internal medicine, pathogenesis, epidemiology, diagnosis, monitoring and treatment protocols. The journal is characterized by the rapid reporting of reviews, original research and clinical studies across all disease areas. The manuscript management system is completely online and includes a very quick and fair peer-review system, which is all easy to use. Visit http://www.dovepress.com/testimonials.php to read real quotes from published authors.

Submit your manuscript here: https://www.dovepress.com/international-journal-of-general-medicine-journal 